\title{
A novel combination of bortezomib, lenalidomide, and clarithromycin produced stringent complete response in refractory multiple myeloma complicated with diabetes mellitus - clinical significance and possible mechanisms: a case report
}

\author{
Nobuo Takemori ${ }^{*}$ (D), Goro Imai ${ }^{2}$, Kazuo Hoshino ${ }^{3}$, Akishi Ooi ${ }^{4}$ and Masaru Kojima ${ }^{5}$
}

\begin{abstract}
Background: In general, dexamethasone is a required component drug in various combination chemotherapies for treating multiple myeloma, and its efficacy has been widely recognized. However, administration of dexamethasone is known to cause various adverse effects including hyperglycemia which requires insulin therapy. During the course of treatment, we developed a novel effective dexamethasone-free combination regimen and evaluated it for its effect in multiple myeloma.

Case presentation: We report a case of 68-year-old Japanese woman with refractory advanced Bence-Jones- $\lambda$ type multiple myeloma associated with diabetes mellitus. Various combination regimens were carried out, but the response to some regimens was insufficient or others containing dexamethasone, although effective, were inappropriate to continue due to aggravation of diabetes mellitus. Thus, we developed a dexamethasone-free, short dosing-period regimen consisting of bortezomib, lenalidomide, and clarithromycin. This regimen was found to be highly effective and succeeded in achieving stringent complete response.

Conclusions: The successful dexamethasone-free regimen clearly shows that dexamethasone is not a requisite component in treating multiple myeloma, and it can be substituted with clarithromycin. This regimen is particularly useful for treating patients with multiple myeloma associated with diabetes mellitus.
\end{abstract}

Keywords: Combination chemotherapy, Clarithromycin, Diabetes mellitus, Refractory multiple myeloma, Stringent complete response

\section{Background}

Lenalidomide (Len; Revlimid ${ }^{\circ}$ ) has been used in combination with dexamethasone (Dex) for treating relapsed/refractory multiple myeloma (MM) [1], newly diagnosed MM [2, 3], and transplant-ineligible MM [4]. Subsequently, bortezomib (Bor; Velcade ${ }^{\circ}$ ), the first-inclass proteasome inhibitor of the $26 \mathrm{~S}$ proteasome, was

\footnotetext{
*Correspondence: takemori_nobuo@circus.ocn.ne.jp

${ }^{1}$ Division of Hematology, Department of Internal Medicine, Imai Hospital,

Tanaka-cho 100, Ashikaga, Tochigi 326-0822, Japan

Full list of author information is available at the end of the article
}

approved in combination with Dex for treating newly diagnosed MM prior to autologous stem cell transplantation [5]. Recently, both drugs have been widely used for treating MM. In general, high-dose/low-dose Dex is a requisite component in various combination chemotherapies for treating $M M$, and its efficacy has been recognized. However, administration of Dex is known to cause various adverse effects including hyperglycemia leading to secondary diabetes mellitus (DM), DM-aggravation, DM-nephropathy, DM-retinopathy, reactivation of hepatitis virus B leading to fulminant hepatitis, 
increased susceptibility to infections, Cushing syndrome, psychic disturbance like delirium, systemic osteoporosis, cataract, and so on. Sometimes, clinicians have to treat patients with MM who also have DM. In such cases, regimens that include Dex induce severe hyperglycemia which requires insulin therapy. We treated a patient with refractory Bence-Jones (BJ)- $\lambda$ type MM associated with DM, and succeeded in achieving stringent complete response (sCR) with a Dex-free, short dosing-period (sdp) regimen in which clarithromycin (CAM) was combined with Bor and Len.

\section{Case presentation}

Our patient was a 68-year-old Japanese woman $(48 \mathrm{~kg} /$ $151 \mathrm{~cm}$ ) with a 7-year history of DM. She had a past history of receiving a radical mastectomy for right mammary cancer in the department of surgery of our hospital at the age of 65 . Her postoperative course was uneventful. After the operation, she received chemotherapies (trastuzumab, paclitaxel, and tegafur-uracil) until the end of December 2014, and later she was followed without treatment on an out-patient basis. In March 2015, she showed progressive unexplained anemia. On 4 June 2015, she was admitted to the surgical department of our hospital at the age of 68 because of anemia, abdominal pain, paralytic ileus, generalized neuralgialike pains, and bilateral shoulder joint swellings and pains. On 13 June 2015, she was referred and admitted to the hematologic division for further evaluation.

Physical examinations on admission were as follows. Her temperature was $36.5^{\circ} \mathrm{C}$, her pulse was 88 , and her blood pressure was 130 systolic and 85 diastolic. Her right mamma was completely removed with a remaining operation scar. She appeared chronically ill and could not turn over due to generalized neuralgia-like pains and severe right shoulder pains. Her skin showed pallor and palpebral conjunctivae were anemic. Her abdomen was distended, tympanitic, and diffusely tender without peristalsis, indicating paralytic ileus. Superficial lymph nodes were impalpable.

Laboratory data on admission were as follows. A complete blood cell count showed moderate anemia (red blood cell count, $2.31 \times 10^{12} / \mathrm{L}$; hemoglobin $7.4 \mathrm{~g} / \mathrm{dL}$ ), slightly increased leukocytes (white blood cell count, $9.5 \times 10^{9} / \mathrm{L}$ with $67.5 \%$ neutrophils, $17.5 \%$ lymphocytes, and $13 \%$ monocytes, $2 \%$ eosinophils) and normal platelet count $\left(301 \times 10^{9} / \mathrm{L}\right)$. Elevated levels of serum free light chain- $\lambda$ (FLC- $\lambda$; more than $3200 \mathrm{mg} / \mathrm{L}$; normal range (nr.), 4.44 26.18 mg/L), $\beta_{2}$-microglobulin (BMG; $6.5 \mathrm{mg} / \mathrm{L}$; nr. $0.9 \sim 1.9 \mathrm{mg} / \mathrm{L}$ ), soluble-interleukin (IL)-2 receptors $(1300 \mathrm{U} / \mathrm{mL}$; nr. 124 466 U/mL), ferritin (955.6 ng/mL; nr. 3.6 114 pg/mL), CRP (1.74 mg/dL; nr. 0 0.26 mg/dL), D-dimer $(8.69 \mu \mathrm{g} / \mathrm{mL}$; $\mathrm{nr} .<0.72 \mu \mathrm{g} / \mathrm{mL})$, and fibrin degradation product (FDP; $20.1 \mu \mathrm{g} / \mathrm{mL}$; nr. < $5 \mu \mathrm{g} / \mathrm{mL}$ ), N-terminal pro-brain natriuretic peptide (NTproBNP; $191 \mathrm{pg} / \mathrm{mL}$; nr. $\leq 125 \mathrm{pg} / \mathrm{mL}$ ), CA19-9 (42 U/ mL; nr. $\leq 37 \mathrm{U} / \mathrm{mL})$, sialyl-Le ${ }^{\mathrm{x}}-\mathrm{i}(\mathrm{SLX} ; 42.8 \mathrm{U} / \mathrm{mL}$; nr. $\leq$ $38 \mathrm{U} / \mathrm{mL}$ ), glycated hemoglobin (HbA1c; 6.7\%; nr. $4.54 \sim 6.25 \%)$ and uric acid (7.2 mg/dL; nr. $2.5 \sim 7.0 \mathrm{mg} /$ $\mathrm{dL}$ ), and depressed levels of immunoglobulin (Ig) G (341 mg/dL; nr. 870 1700 mg/dL), IgA (15 mg/dL; nr. $110 \sim 410 \mathrm{mg} / \mathrm{dL}), \operatorname{IgM}(10 \mathrm{mg} / \mathrm{dL} ; \mathrm{nr} .46 \sim 260 \mathrm{mg} / \mathrm{dL})$, IgD $(<1.0 \mathrm{mg} / \mathrm{dL} ; \mathrm{nr} . \leq 12.6 \mathrm{mg} / \mathrm{dL})$, total protein $(6.4 \mathrm{~g} /$ $\mathrm{dL}$; nr. 6.7 8.3 g/dL), albumin (3.8 g/dL; nr. 3.9 4.9 g/ $\mathrm{dL}$ ), zinc turbidity test (ZTT; $1 \mathrm{KU} ; \mathrm{nr} .4 \sim 12 \mathrm{KU})$, serum Fe $(50 \mu \mathrm{g} / \mathrm{dL} ; \mathrm{nr} .54 \sim 181 \mu \mathrm{g} / \mathrm{dL})$ and total cholesterol (106 mg/dL; nr. 130 219 mg/dL) were observed. Values of carcinoembryonic antigen (CEA), aspartate aminotransferase (AST), alanine aminotransferase (ALT), lactate dehydrogenase (LDH), creatine phosphokinase (CPK), amylase, blood urea nitrogen (BUN), creatinine, and electrolytes were within normal range. Titers of rheumatoid arthritis particle agglutination, anti-nuclear antibody, anti-microsome antibody, and antithyroglobulin antibody were within normal range. Her urine showed proteinuria (7.4 g/day). Lambda-type BJ protein was detected in her urine by immunofixation electrophoresis. Bone scintiscan using ${ }^{99 \mathrm{~m}}$ Tc-hydroxymethylene diphosphonate (HMDP) revealed multiple uptakes of radioisotope in generalized bones, particularly in vertebrae and shoulder joints. Chest and abdominal computed tomography scans revealed massive soft tissue masses around bilateral shoulder joints suggestive of amyloid light-chain (AL) deposition (shoulder-pad sign), paralytic ileus, small multiple mesenteric lymph nodes, small amount of ascites, slight right hydronephrosis, and slight splenomegaly. Skull X-ray films showed sporadic punched out lesions.

An iliac bone marrow (BM) puncture performed on 16 June 2015 showed that her BM was infiltrated by atypical medium-sized and large-sized myeloma cells with basophilic cytoplasm and fine chromatin networks with occasional nucleoli. Binuclear and trinuclear myeloma cells were frequently seen (Fig. 1). Myeloma cells accounted for $70.8 \%$ of total nucleated cells on smear preparation. On immunohistochemical examination, they were exclusively positive for light chain $\lambda$.

A chromosomal analysis of the BM cells revealed complex hyperdiploidies (Fig. 2). Thus, a diagnosis of $\mathrm{BJ}-\lambda$ type MM (Durie and Salmon, stage IIIA; International Staging System, stage III; Southwest Oncology Group, stage III) was made.

In the hematologic division of our hospital, most of the patients with $\mathrm{MM}$ are vulnerable older people with severe complications. Thus, the dose of Len is minimized to $10 \mathrm{mg} /$ day in those patients to avoid the adverse effects, such as leukopenia, thrombocytopenia, 


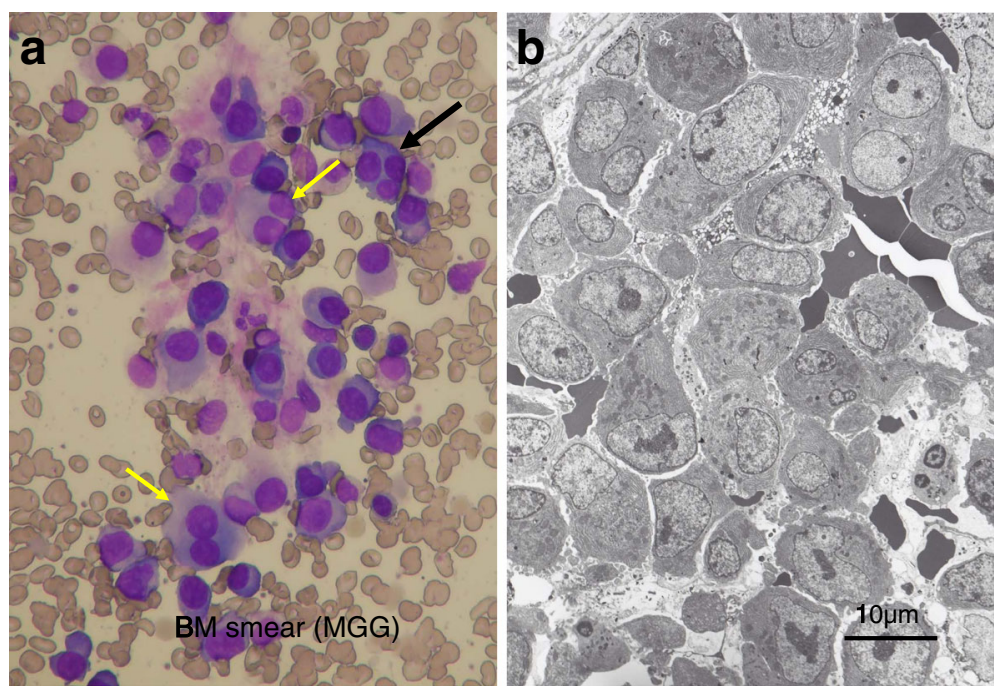

Fig. 1 Bone marrow obtained before the initiation of treatment. a Bone marrow smear. Bone marrow is infiltrated by immature myeloma cells showing basophilic cytoplasm and fine nuclear chromatin networks with occasional nucleoli. Binuclear (yellow arrows) and trinuclear (black arrow) myeloma cells, suggesting hyperdiploidies, are seen (under $\times 40$ magnification objective). b Electron micrograph of the bone marrow. Bone marrow is compactly occupied by immature myeloma cells with remarkable nucleoli, fine chromatin networks, and abundant rough endoplasmic reticula

toxicoderma, deep venous thrombosis, neuropathy, a high fever associated with elevated CRP (that is, transient inflammatory reaction), and so on. In fact, $10 \mathrm{mg}$ of Len was found to be effective enough for treating MM with reduced adverse effects, and allowing for safe repetitions of treatments [6]. Moreover, doses of Bor and
Dex are also reduced to improve tolerability and to optimize efficacy as suggested by Palumbo et al. [7].

The whole clinical course (Fig. 3), the detailed clinical course (Fig. 4), and regimens used during the whole clinical course are listed in Table 1. At first, three cycles of (1) vincristine, adriamycin, and Dex

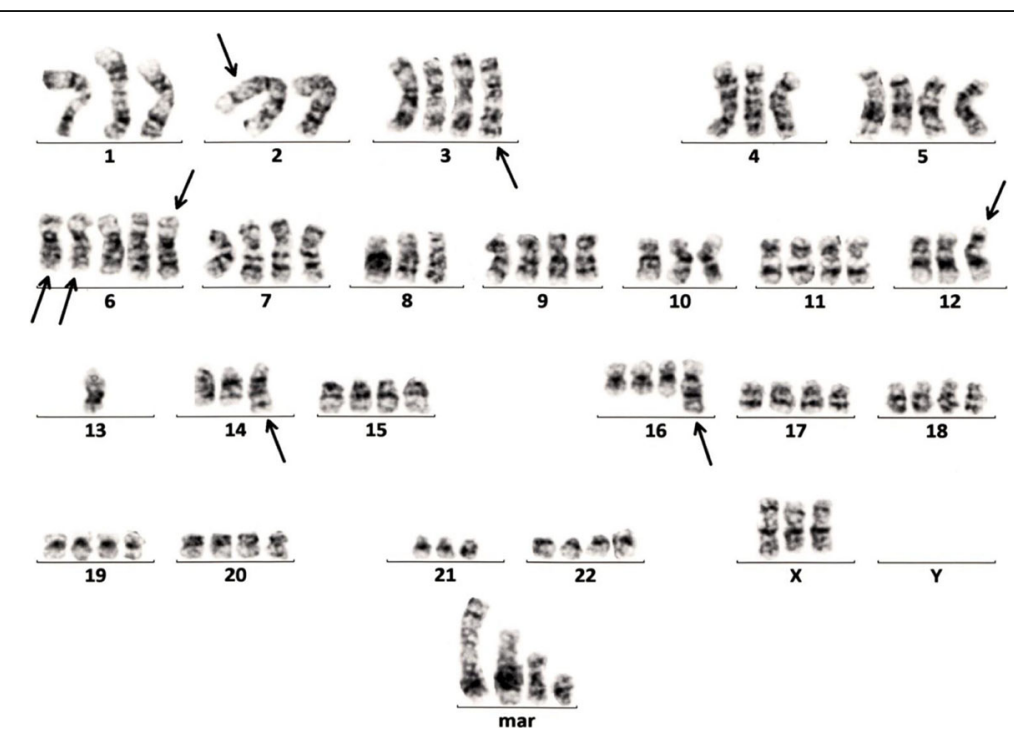

83 84, XXX, -X, -1, -2, -2, add (2) (p13), add (3) (q27), -4, +6, del (6) (q?) $\times 2,-8$, $-10,-12$, add (12) (p11.2), -13, -13, -13, -14, add (14) (q32), der (16), t (1;16) (q21; q12-13), $-21,+4 \sim 8 \operatorname{mar}[3 / 10]$ 46, XX [7/10] Arrows indicate chromosomes involving add, del, der and translocation.

Fig. 2 Chromosome analysis showing complex hyperdiploidies. Hyperdiploidies were seen in three out of ten cells (3/10). Seven out of ten cells showed a normal karyotype (7/10). Similar hyperdiploidies remained until achieving stringent complete response 


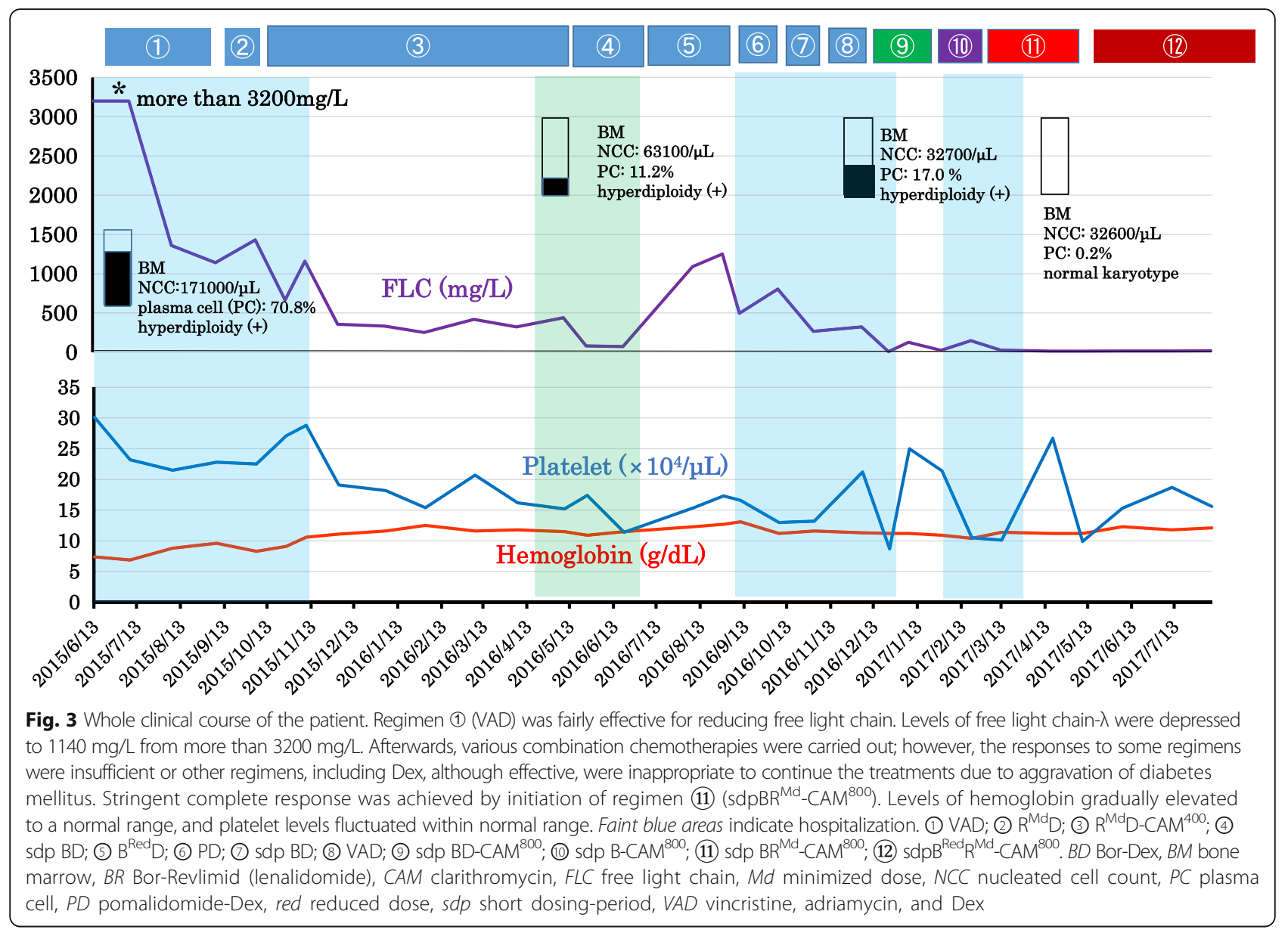

(VAD) therapy were carried out. This treatment was fairly effective; levels of FLC- $\lambda$ and BMG were depressed to $1140 \mu \mathrm{g} / \mathrm{L}$ from more than $3200 \mu \mathrm{g} / \mathrm{L}$ and to $2.2 \mathrm{mg} / \mathrm{L}$ from $6.5 \mathrm{mg} / \mathrm{L}$, respectively. Bilateral shoulder joint pains and paralytic ileus were rapidly improved. She was released from confinement to bed and able to walk 3 weeks after starting the first cycle of VAD therapy. Although this regimen proved to be effective for relieving her symptoms, levels of FLC- $\lambda$ still remained high $(1140 \mathrm{mg} / \mathrm{L})$. As shown in Table 1 and Fig. 3, regimens (1) (12) were consecutively carried out. In general, the regimens including Dex caused severe hyperglycemia which required insulin therapy, and most of them, except regimen (3) in which CAM (400 $\mathrm{mg} /$ day for 3 weeks) was combined with Len and Dex, could not be efficiently repeated due to DM aggravation or inefficacy of the regimens. Of all the regimens, regimen (9) (short dosing-period [sdp] BorDex-CAM ${ }^{800}$ ), and the regimen (11)/12) ( $\mathrm{sdpB}^{\text {(Reduced }}$ dose $[\mathrm{Rd}] \mathrm{R}^{\text {Minimized dose }[\mathrm{Md}]-\mathrm{CAM}^{800}}$ proved to be very effective. Levels of FLC- $\lambda$ were drastically depressed to a normal range by regimens (9) and (11) (Fig. 4).
However, the former could not be repeated because of the aggravation of $\mathrm{DM}$, leading to deterioration of the general condition of our patient. On the other hand, regimen (11) could be safely repeated without fearing DM aggravation; the levels of FLC- $\lambda$ were brought down to a normal range $(12.9 \mathrm{mg} / \mathrm{L})$ with normal $\mathrm{\kappa} / \lambda$ ratio (1.024) after four cycles of this regimen (Table 1). Myeloma cells disappeared from her BM (Figs. 3 and 4) and BJ proteins disappeared from her serum and urine. Two color flow cytometric analysis of the BM showed absence of clonal plasma cells (PCs). In addition, levels of serum $\operatorname{IgG}, \operatorname{IgA}$, and $\operatorname{IgM}$ elevated to normal levels. Thus, our patient was proved to be in SCR according to the updated criteria of International Myeloma Working Group (IMWG) [8]. In May 2017, urticaria started to appear after subcutaneous (sc) injections of Bor. Thus, the administration of Bor was reduced to once a week from twice a week (regimen (12): $\mathrm{sdpB}^{\mathrm{Red}} \mathrm{R}^{\mathrm{Md}}-\mathrm{CAM}^{800}$ ) and intravenous administration of hydrocortisone $100 \mathrm{mg}$, prior to each Bor injection, was started to prevent urticaria. Regimen (12) was safely repeated without fearing DM aggravation as well as 


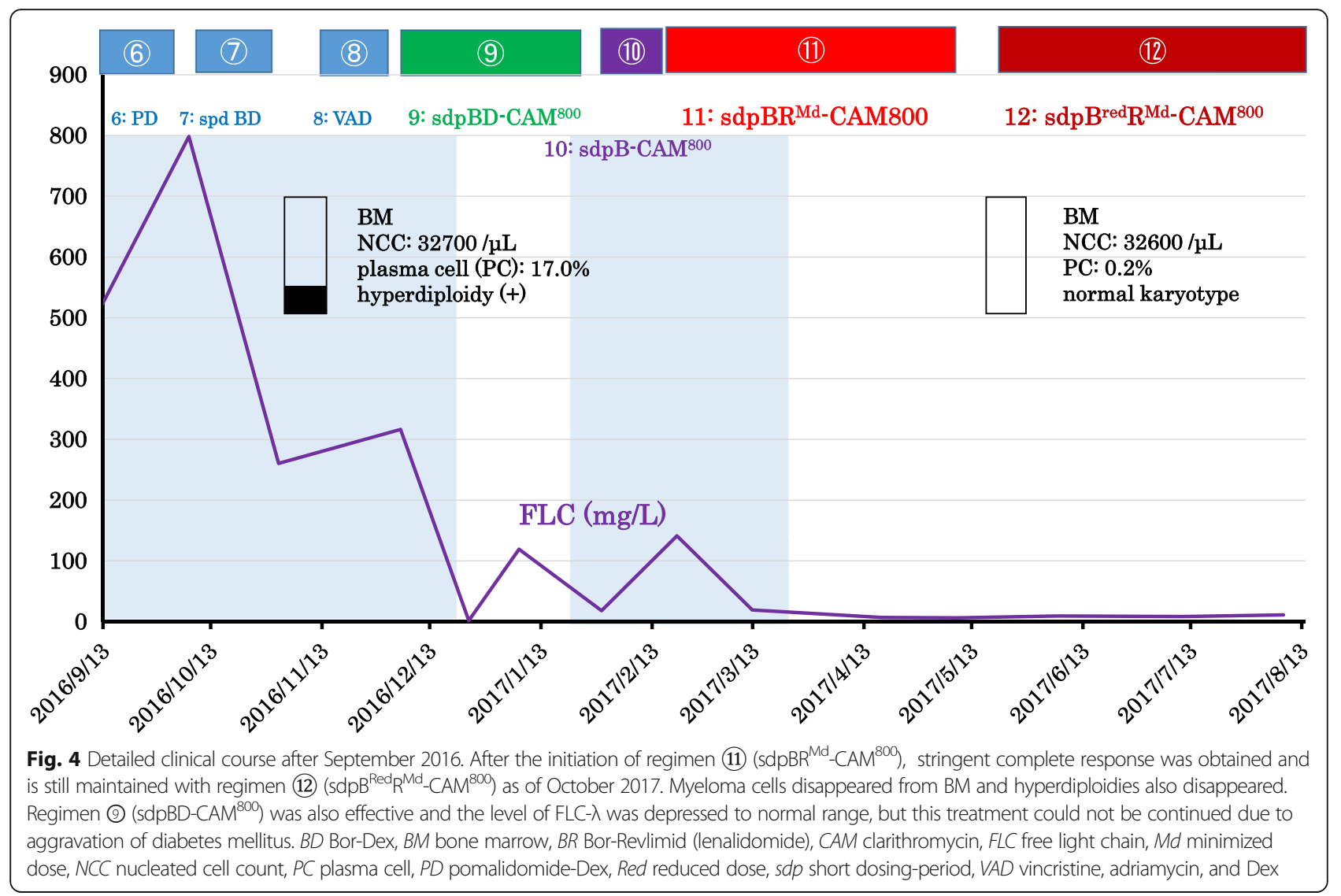

urticaria. Levels of FLC- $\lambda$ remained within normal range, and our patient is in good condition as of November 2017.

\section{Discussion}

It is important to elucidate the mechanisms whereby $\mathrm{SCR}$ is achieved by the combination regimen consisting of Len, Bor and CAM. Bor, the 26S proteasome inhibitor, has been widely used for treating MM [9]. Increasing lines of evidence indicate that inhibition of the $26 \mathrm{~S}$ proteasome by Bor, that is, blocking of the ubiquitin proteasome system by Bor, leads to the accumulation of unfolded or misfolded protein in the endoplasmic reticulum (ER) in myeloma cells; this results in ER stress followed by a coordinated cellular response known as unfolded protein response (UPR) [10-13]. UPR is known to induce activation of the chaperone protein GRP78 (Bip) to maintain ER integrity and upregulates transcription factor $\mathrm{CHOP}$ (the $\mathrm{C} / \mathrm{EBP}$ homologous protein, also designated as GADD153) to mediate cell death when ER stress is beyond the tolerance of the cell adaptation [1013]. In the clinical field, Bor is now widely used in combination regimens such as VMP (Bor, melphalan, and prednisolone), VTD (Bor, thalidomide, and Dex), PVD (Pomalidomide, Bor, and Dex), or BRD (Bor, Len, and Dex) in refractory/relapsed MM or newly diagnosed
MM. Although regimens involving Bor and Dex have contributed to substantial improvement of MM, the treatment of patients with MM associated with DM is still a troublesome issue, and therapeutic improvements are required.

As for CAM, many investigators have reported its immunosuppressive or immunomodulatory effects in patients with cancers or tumor-bearing animals. In brief, CAM is known to decrease the production of IL-1, IL-2, IL-5, IL-6, IL-8, IL-10, tumor necrosis factor (TNF)- $\alpha$, transforming growth factor (TGF)- $\alpha, \mathrm{TGF}-\beta$, and matrix metalloproteinase 9 , and increase the production of IL-4, IL-12, and interferon- $\gamma[6,14,15]$. In addition, CAM is known to induce apoptosis through Fas-Fas ligand pathway [16].

Recently, the high efficacy of the chemotherapeutic regimen combining CAM (Biaxin ${ }^{\odot}$ ) with Len and Dex (BiRD regimen) in treating $\mathrm{MM}$ has been documented $[17,18]$. Concerning the role of CAM in BiRD therapy, the steroid-enhancing/sparing effect is suggested [6, 14, 17, 19]; however, the exact mechanisms of action of CAM remain uncertain. Nakamura et al. [20] reported that CAM attenuates autophagy and induces cell growth inhibition in MM cells; more exactly, the treatment with CAM attenuates autophagy by blocking the late phase of the autophagic process, probably after 
Table 1 Brief summary of regimens used during the whole clinical course

\begin{tabular}{|c|c|c|c|c|}
\hline Regimen & Cycles & Date of treatments & $\mathrm{FLC}-\lambda(\mathrm{mg} / \mathrm{L}) / \mathrm{BMG}(\mathrm{mg} / \mathrm{L})$ & Evaluation \\
\hline (1) VAD & 3 & Jun. 19 Sep.5/2015 & $\geq 3200 \rightarrow 1140 / 6.5 \rightarrow 2.2$ & Effective \\
\hline (2) $R^{M d} D$ & 1 & Sep.14 Oct. 4/2015 & $1140 \rightarrow 1430 / 2.2 \rightarrow 2.5$ & Ineffective \\
\hline (3) $R^{M d} D-C A M M^{400}$ & 7 & Oct. 12 May 15/2016 & $1430 \rightarrow 439 / 2.5 \rightarrow 1.5$ & Effective \\
\hline (4) $s d p ~ B D$ & 2 & May 16 Jun. 29/2016 & $439 \rightarrow 72.9 / 1.5 \rightarrow 1.3$ & Effective \\
\hline (5) $B^{\text {Red } D}$ & 2 & Jul. 11 Sep. 6/2016 & $72.9 \rightarrow 496 / 1.3 \rightarrow 1.5$ & Ineffective \\
\hline (6) PD & 1 & Sep. 13 Oct. 3/2016 & $496 \rightarrow 805 / 1.5 \rightarrow 1.9$ & Ineffective \\
\hline (7) $s d p B D$ & 1 & Oct. 17 Oct. 30/2016 & $805 \rightarrow 267 / 1.9 \rightarrow 1.6$ & Fairly effective \\
\hline (8) VAD & 1 & Nov. 14 Dec. 6/2016 & $267 \rightarrow 323 / 1.6 \rightarrow 1.4$ & Ineffective \\
\hline (9) $s d p B D-C A M{ }^{800}$ & 2 & Dec. 12 Jan. 23/2017 & $323 \rightarrow 24.9 / 1.4 \rightarrow 1.1$ & Very effective \\
\hline (10) $s d p B-C A M^{800}$ & 1 & Feb. 4 Feb. 26/2017 & $24.9 \rightarrow 148 / 1.1 \rightarrow 2.1$ & Ineffective \\
\hline (11) $s d p B R^{M d}-c^{80 m} 800$ & 4 & Feb. 27 May 8/2017 & $148 \rightarrow 12.9 / 2.8 \rightarrow 2.1$ & Very effective \\
\hline (12) $s d p B^{\text {Red }} R^{M d}-C A M^{800}$ & 6 & May 23 Oct. 31/2017 & $12.9 \rightarrow 14.2 / 2.1 \rightarrow 1.4$ & Effective/stable \\
\hline
\end{tabular}

$A$ (ADM) adriamycin, $B$ bortezomib, BMG ß2-microglobulin, CAM clarithromycin, $D$ (Dex) dexamethasone, $F L C$ free light chain, $M d$ minimized-dose, $P$ (Pom) pomalidomide, $R$ Revlimid (Len: lenalidomide), Red reduced dose, sc subcutaneous, sdp short dosing-period, $V$ vincristine, $w k$ week

(1) VAD: V $0.4 \mathrm{mg} /$ body·ADM $9 \mathrm{mg} / \mathrm{m}^{2}$, continuous intravenous infusion, on days 1-4; Dex $40 \mathrm{mg} / \mathrm{body}$, on days $1-4,9-12,17-20$, every 4-wk cycle

(2) $\mathrm{R}^{\mathrm{Md}} \mathrm{D}$ : Len $10 \mathrm{mg} /$ day for $3 \mathrm{wks}$; Dex $20 \mathrm{mg} /$ day, twice/week for 3 wks, every 4-wk cycle

(3) $R^{M d} D-C A M{ }^{400}: R^{M d} D$ combined with CAM $400 \mathrm{mg} /$ day, $200 \mathrm{mg}$ twice/day for 3 wks, every 4-wk cycle

(4) sdpBD: Bor $1.3 \mathrm{mg} / \mathrm{m}^{2}$, sc, twice/wk for $2 \mathrm{wks}$; Dex $20 \mathrm{mg} /$ day twice/wk for $2 \mathrm{wks}$, every 4-wk cycle

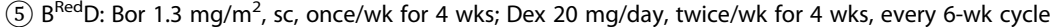

(6) PD: Pom $4 \mathrm{mg} / \mathrm{day}$ for 3 wks; Dex $20 \mathrm{mg} /$ day twice/wk for 3 wks, every 4-wks cycle

(7) sdpBD (8VAD

9) sdpBD-CAM ${ }^{800}$ : sdpBD combined with CAM $800 \mathrm{mg} /$ day, $400 \mathrm{mg}$ twice/day for 2 wks, every 4-wk cycle

(10) sdpB-CAM ${ }^{800}$ : Bor $1.3 \mathrm{mg} / \mathrm{m}^{2}$, sc, twice/wk for $2 \mathrm{wk}$; CAM $800 \mathrm{mg} /$ day, $400 \mathrm{mg}$ twice/day for 2 wks, every 4-wk cycle

(11) $\mathrm{sdpBR}^{\mathrm{Md}}$-CAM ${ }^{800}$ : Bor $1.3 \mathrm{mg} / \mathrm{m}^{2}$, sc, twice/wk for 2 wks; Len $10 \mathrm{mg} /$ day for 2 wks; CAM $800 \mathrm{mg} /$ day, $400 \mathrm{mg}$ twice/day for 2 wks, every 4 -wk cycle

(12) $\mathrm{sdpB}^{\text {Red }} \mathrm{R}^{\mathrm{Md}}-\mathrm{CAM}^{800}$ : Bor $1.3 \mathrm{mg} / \mathrm{m}^{2}$, sc, once/wk for 2 wks; Len $10 \mathrm{mg} /$ day for 2 wks; CAM $800 \mathrm{mg} /$ day, $400 \mathrm{mg}$ twice/day for 2 wks, every $4-\mathrm{wk}$ cycle

the fusion of autophagosomes with lysosomes in myeloma cells.

Although CAM by itself exhibits no cytotoxicity $[6,12$, 21], simultaneous inhibition of the ubiquitin-proteasome system by Bor and autophagy-lysosome system by CAM will synergistically lead to activate UPR, resulting in enhanced MM cell apoptosis [12, 22].

Since MM is characterized by uncontrolled cell growth of monoclonal antibody-producing neoplastic PCs, large quantities of unfolded or misfolded Ig production itself triggers ER stress [22]. This may mean that MM is a specific neoplasm susceptible to proteasome and autophagy inhibitors. In support of this proposal, Obeng et al. [11] reported that MM cells have a lower threshold for proteasome inhibitor-induced UPR induction and ER stress-induced apoptosis.

In BiRD regimen, CAM at a dose of $1000 \mathrm{mg} /$ day, $500 \mathrm{mg}$ twice a day, was recommended $[17,18]$. In the present case, we applied a relatively high dose of CAM $(800 \mathrm{mg} /$ day, $400 \mathrm{mg}$ twice a day, for 2 weeks) in regimens (9) (12), and succeeded in achieving and maintaining sCR by the regimen (11//12) without any severe adverse effects.

Len has generally been used in combination with Dex for treating refractory and relapsed MM [1], newly diagnosed MM [2, 3], and transplant-ineligible MM [4]. The precise cellular targets and the exact mechanisms of action of Len remain unclear. However, recent studies revealed that Len's multiple effects include: (1) immune modulation (CD4+ and CD8+ T cell co-stimulation, regulatory $\mathrm{T}$ cell suppression, Th1 cytokine production, NK and NKT cell activation, and antibody-dependent cellular cytotoxicity), (2) interference with tumor microenvironment interactions (anti-angiogenesis, antiinflammatory properties, downregulation of adhesion molecules, and anti-osteoclastogenic properties) and (3) direct anti-tumor effects (inhibition of cyclin-dependent kinase, induced expression of tumor suppressor genes such as Egr-1, Egr-2, and SPARC genes, downregulation of NF- $\mathrm{kB}$, and inhibition of caspase 3,8 , and 9) [23]. Len is known to suppress TNF- $\alpha$, IL-1 $\beta$, IL-6, IL-12, TGF- $\beta$, macrophage inhibitory protein- $\alpha$, granulocytemacrophage colony-stimulating factor, insulin-like growth factor-1, basic FGF, RANKL, and vascular endothelial growth factor (VEGF), and to increase IL-2, IL-10, and interferon- $\gamma$. Inhibition of VEGF by Len may alter the BM microvasculature, thereby making the microenvironment less hospitable for MM cell growth [6, 22-25]. Recently, Krönke et al. [26] demonstrated that Len causes selective ubiquitination and degradation of two lymphoid transcription factors - Ikaros family zinc finger proteins (IKZF) 1 and 3 (IKZF1, Ikaros; and IKZF3, Aiolos) - by the CRBNCRL4 ubiquitin ligase. It is known that IKZF1 and IKZF3 are essential B cell transcription factors. A study in mice demonstrated that IKZF3 is required for the generation of 
PCs [27]. In this connection, Lu et al. [28] reported that Len-bound cereblon acquires the ability to target IKZF1 and IKZF3 for proteasomal degradation in MM cells. They analyzed myeloma cell lines and demonstrated that loss of IKZF1 and IKZF3 is necessary and sufficient for Len's therapeutic effect. In the present case, we demonstrated that regimen (11)/12) $\left(\mathrm{sdpBR}^{\mathrm{Md}}-\mathrm{CAM}^{800}\right.$ or $\mathrm{sdpB}^{\mathrm{Red}} \mathrm{R}^{\mathrm{Md}}$ $\mathrm{CAM}^{800}$ ) is highly effective for treating MM. Possibly, Len's diverse apoptosis-inducing effects might further enhance cytotoxic effects of Bor and CAM.

In general, Dex is considered to be a requisite for treating $\mathrm{MM}$, and it is included in most of the regimens for MM. However, in the present case, sCR was easily achieved by a Dex-free regimen $\left(\operatorname{sdpBR}{ }^{\mathrm{Md}}-\mathrm{CAM}^{800}\right.$ or $\mathrm{sdpB}^{\mathrm{Red}} \mathrm{R}^{\mathrm{Md}}$ $\left.\mathrm{CAM}^{800}\right)$. This seems to indicate that Dex is not necessarily requisite in treating MM. Nevertheless, the effect of regimen (9) (sdpBD-CAM ${ }^{800}$ ), which includes Dex, was equal to regimen (11) $\left(\mathrm{sdpBR}^{\mathrm{Md}}-\mathrm{CAM}^{800}\right)$, but the former was discontinued because of severe hyperglycemia which required insulin therapy. In contrast, the effect of Lenomitted regimen (10) (sdp B-CAM ${ }^{800}$ ) was ineffective. This means that the combination of three components, namely, Bor, Len, and CAM are requisite for treating MM.

It is well known that CAM is the potent CYP3A4 inhibitor and Bor is metabolized by CYP3A4. Therefore, concomitant administration of Bor and CAM may lead to intracellular elevation of Bor concentrations [12]. One may ask whether the enhanced cytotoxicity achieved by regimens including Bor and CAM is simply due to the pharmaco-interaction between Bor and CAM via CYP3A4. We cannot completely exclude this possibility.

\section{Conclusions}

In the present study, we clearly demonstrated that sdpBR ${ }^{\mathrm{Md}}-\mathrm{CAM}^{800}$ or $\mathrm{sdpB}^{\mathrm{Red}} \mathrm{R}^{\mathrm{Md}}-\mathrm{CAM}^{800}$ regimen is highly effective for treating refractory MM. This regimen has outstanding merits as follows. (1) The regimen is Dex-free, therefore there is no risk of developing steroidinduced DM/DM aggravation or DM-related complications. (2) The regimen is based on a dose-reduction schedule, the adverse effects of Bor and Len are attenuated, and its medical cost is less expensive. (3) The regimen is based on a short-term schedule; it is less risky in developing various adverse effects and gives patients enough time to recover, enabling clinicians to repeat it safely. (4) The regimen contains CAM which might play an important role in enhancing synergistically the effects of Bor and Len. The $\mathrm{sdpB}^{(\mathrm{Red})} \mathrm{R}^{\mathrm{Md}}-\mathrm{CAM}^{800}$ regimen is a promising combination therapy for patients with MM particularly associated with DM.

\section{Abbreviations}

BD: Bortezomib-dexamethasone; BJ: Bence-Jones; BM: Bone marrow; BMG: $\beta_{2}$ microglobulin; Bor: Bortezomib; CAM: Clarithromycin; Dex: Dexamethasone; DM: Diabetes mellitus; ER: Endoplasmic reticulum; FLC: Free light chain;
Ig: Immunoglobulin; IKZF: Ikaros family zinc finger proteins; IL: Interleukin; Len: Lenalidomide; MM: Multiple myeloma; nr.: Normal range; PC: Plasma cell; sCR: Stringent complete response; sdp: Short dosing-period;

TGF: Transforming growth factor; TNF: Tumor necrosis factor; UPR: Unfolded protein response; VAD: Vincristine, adriamycin, and dexamethasone;

VEGF: vascular endothelial growth factor

\section{Acknowledgements}

The authors would like to thank Prof. Hong-Kean Ooi (Department of Veterinary Medicine, Azabu University) in helpful reviewing and preparing the manuscript.

\section{Funding}

This study was not supported by any grant.

Availability of data and materials

Not applicable

\section{Authors' contributions}

NT carried out the clinical treatment, follow-up, and collecting data, and was also a major contributor in writing the manuscript. Gl assisted in treating the patient. KH performed the mastectomy and postoperative follow-up. AO and MK performed the immunohistochemistry and were major contributors for the pathological diagnosis. All authors read and approved the final manuscript.

\section{Ethics approval and consent to participate}

The patient was fully informed of the risk of modified treatments and the theoretical basis of the treatments for MM was explained to her.

\section{Consent for publication}

Written informed consent was obtained from the patient for publication of this case report and any accompanying images. A copy of the written consent is available for review by the Editor-in Chief of this journal.

\section{Competing interests}

The authors declare that they have no competing interests.

\section{Publisher's Note}

Springer Nature remains neutral with regard to jurisdictional claims in published map and institutional affiliations.

\section{Author details}

${ }^{1}$ Division of Hematology, Department of Internal Medicine, Imai Hospital, Tanaka-cho 100, Ashikaga, Tochigi 326-0822, Japan. ²Department of Internal Medicine, Imai Hospital, Tanaka-cho 100, Ashikaga, Tochigi 326-0822, Japan. ${ }^{3}$ Department of Surgery, Imai Hospital, Tanaka-cho 100, Ashikaga, Tochigi 326-0822, Japan. ${ }^{4}$ Department of Molecular and Cellular Pathology, School of Medicine, Kanazawa University, 13-1 Takara-machi, Kanazawa, Ishikawa 920-8640, Japan. ${ }^{5}$ Department of Diagnostic Pathology, Dokkyo Medical University School of Medicine, Shimotsuga-gun, Mibu-machi, Tochigi 321-0293, Japan.

Received: 30 October 2017 Accepted: 21 December 2017

Published online: 18 February 2018

\section{References}

1. Dimopoulos M, Spencer A, Attal M, Prince HM, Harousseau J-L, Dmoszynska A, et al. Lenalidomide plus dexamethasone for relapsed or refractory multiple myeloma. N Engl J Med. 2007;357(21):2123-32.

2. Rajkumar SV, Hayman SR, Lacy MQ, Dispenzieri A, Geyer SM, Kabat B, et al. Combination therapy with lenalidomide plus dexamethasone (Rev/Dex) for newly diagnosed myeloma. Blood. 2005;106(13):4050-3.

3. Rajkumar SV, Jacobus S, Callander NS, Fonseca R, Vesole DH, Williams ME, et al. Lenalidomide plus high-dose dexamethasone versus lenalidomide plus low-dose dexamethasone as initial therapy for newly diagnosed multiple myeloma: an open-label randomised controlled trial. Lancet Oncol. 2010;11: 29-37.

4. Benboubker L, Dimopoulos MA, Dispenzieri A, Catalano J, Belch AR, Cavo M, et al. Lenalidomide and dexamethasone in transplant-ineligible patients with myeloma. N Engl J Med. 2014;371:906-17.

5. Harousseau JL, Attal M, Avet-Loiseau H, Marit G, Caillot D, Mohty M, et al. Bortezomib plus dexamethasone is superior to vincristine plus doxorubicin 
plus dexamethasone as induction treatment prior to autologous stem-cell transplantation in newly diagnosed multiple myeloma: results of the IFM 2005-01 phase III trial. J Clin Oncol. 2010;28(30):4621-9.

6. Takemori N, Fukuda T, Kojima M. Follow-up study of a multiple myeloma patient successfully treated with clarithromycin (CAM), low-dose lenalidomide and low-dose dexamethasone: significance and possible mechanism of action of CAM as an add-on therapy. Case Rep Clin Med. 2014:3:674-84

7. Palumbo A, Bringhen S, Ludwig H, Dimopoulos MA, Bladé J, Mateos MV, et al. Personalized therapy in multiple myeloma according to patient age and vulnerability: a report of the European Myeloma Network (EMN). Blood. 2011;118(17):4519-29.

8. Palumbo A, Rajkumar SV, San Miguel JF, Larocca A, Niesvizky R, Morgan G, et al. International Myeloma Working Group consensus statement for the management, treatment, and supportive care of patients with myeloma not eligible for standard autologous stem-cell transplantation. J Clin Oncol. 2014:32(6):587-600.

9. Chen D, Frezza M, Schmitt S, Kanwar J, Dou QP. Bortezomib as the first proteasome inhibitor anticancer drug: current status and future perspectives. Curr Cancer Drug Targets. 2011;11(3):239-53.

10. Moriya S, Komatsu S, Yamasaki K, Kawai Y, Kokuba H, Hirota A, et al. Targeting the integrated networks of aggresome formation, proteasome, and autophagy potentiates ER stress-mediated cell death in multiple myeloma cells. Int J Oncol. 2015;46(2):474-86.

11. Obeng EA, Carison LM, Gutman DM, Harrington Jr WJ, Lee KP, Boise LH. Proteasome inhibitors induce a terminal unfolded protein response in multiple myeloma cells. Blood. 2006;107(12):4907-16.

12. Komatsu S, Miyazawa K, Moriya S, Takase A, Naito M, Inazu M, et al. Clarithromycin enhances bortezomib-induced cytotoxicity via endoplasmic reticulum stress-mediated CHOP (GADD153) induction and autophagy in breast cancer cells. Int J Oncol. 2012;40(4):1029-39.

13. Verfaillie T, Salazar M, Velasco G, Agostinis P. Linking ER stress to autophagy: potential implications for cancer therapy. Int J Cell Biol. 2010;2010:930509.

14. Takemori N, Kaneko H, Fukuda T, Kojima M. Follow-up study of Epstein-Barr virus-associated Hodgkin's lymphoma (HL) developed in a patient with rheumatoid arthritis (RA) and analysis of circulating cytokines and clinical parameters: clarithromycin coupled with prednisolone is effective for preventing relapse of HL and controlling RA. Arch Clin Microbiol. 2015;6(4):6.

15. Van Nuffel AM, Sukhatme V, Pantziarka P, Meheus L, Sukhatme VP, Bouche G. Repurposing drugs in oncology (ReDO)-clarithromycin as an anti-cancer agent. Ecancer. 2015;9:513

16. Ishimatsu Y, Kadota J, Iwashita T, Nagata T, Ishii H, Shikuwa C, et al. Macrolide antibiotics induce apoptosis of human peripheral lymphocytes in vitro. Int J Antimicrobial Agents. 2004;24:247-53.

17. Niesvizky R, Jayabalan DS, Christos PJ, Furst JR, Naib T, Ely S, et al. BiRD (Biaxin [clarithromycin]/Revlimid [lenalidomide]/dexamethasone) combination therapy results in high complete- and overall-response rates in treatment-naive symptomatic multiple myeloma. Blood. 2008;111(3):1101-9.

18. Gay F, Rajkumar SV, Coleman M, Kumar S, Mark T, Dispenzieri A, et al. Clarithromycin (Biaxin)-lenalidomide-low-dose dexamethasone (BiRd) versus lenalidomide-low-dose dexamethasone (Rd) for newly diagnosed myeloma. Am J Hematol. 2010;85:664-9.

19. Fost DA, Leung DYM, Martin RJ, Brown EE, Szefler SJ, Spahn JD. Inhibition of methylprednisolone elimination in the presence of clarithromycin therapy. J Allergy Clin Immunol. 1999;103:1031-5.

20. Nakamura M, Kikukawa Y, Takeya M, Mitsuya H, Hata H. Clarithromycin attenuates autophagy in myeloma cells. Int J Oncol. 2010;37:815-20.

21. Moreau P, Huynh A, Facon T, Bouilly I, Sotto JJ, Legros L, et al. Lack of efficacy of clarithromycin in advanced multiple myeloma. Leukemia. 1999;13(3):490-1.

22. Moriya S, Che XF, Komatsu S, Abe A, Kawaguchi T, Gotoh A, et al. Macrolide antibiotics block autophagy flux and sensitize to bortezomib via endoplasmic reticulum stress-mediated $\mathrm{CHOP}$ induction in myeloma cells. Int J Oncol. 2013;42(5):1541-50.

23. Quach H, Ritchie D, Stewart AK, Neeson P, Harrison S, Smyth MJ, et al. Mechanism of action of immunomodulatory drugs (IMiDS) in multiple myeloma. Leukemia. 2010;24:22-32.

24. Vallet S, Palumbo A, Raje N, Boccadoro M, Anderson KC. Thalidomide and lenalidomide: mechanism-based potential drug combinations. Leuk Lymphoma. 2008;49(7):1238-45.
25. Richardson P, Mitsiades C, Laubach J, Schlossman R, Ghobrial I, Hideshima T, et al. Lenalidomide in multiple myeloma: an evidence-based review of its role in therapy. Core Evid. 2009;4:215-45.

26. Krönke J, Udeshi ND, Narla A, Grauman P, Hurst SN, McConkey M, et al. Lenalidomide causes selective degradation of IKZF1 and IKZF3 in multiple myeloma cells. Science. 2014;343(6168):301-5.

27. Cortés M, Georgopoulos K. Aiolos is required for the generation of high affinity bone marrow plasma cells responsible for long-term immunity. J Exp Med. 2004;199(2):209-19.

28. Lu G, Middleton RE, Sun H, Naniong M, Ott CJ, Mitsiades CS, et al. The myeloma drug lenalidomide promotes the cereblon-dependent destruction of Ikaros proteins. Science. 2014;343:305-9.

\section{Submit your next manuscript to BioMed Central and we will help you at every step:}

- We accept pre-submission inquiries

- Our selector tool helps you to find the most relevant journal

- We provide round the clock customer support

- Convenient online submission

- Thorough peer review

- Inclusion in PubMed and all major indexing services

- Maximum visibility for your research

Submit your manuscript at www.biomedcentral.com/submit
Biomed Central 